\title{
Categorified Symplectic Geometry and the Classical String
}

\author{
John C. Baez, Alexander E. Hoffnung, Christopher L. Rogers \\ Department of Mathematics, University of California, Riverside, California 92521, \\ USA.E-mail: baez@math.ucr.edu; alex@math.ucr.edu; chris@math.ucr.edu
}

Received: 13 September 2008 / Accepted: 19 August 2009

Published online: 20 November 2009 - (C) The Author(s) 2009. This article is published with open access at Springerlink.com

\begin{abstract}
A Lie 2-algebra is a 'categorified' version of a Lie algebra: that is, a category equipped with structures analogous to those of a Lie algebra, for which the usual laws hold up to isomorphism. In the classical mechanics of point particles, the phase space is often a symplectic manifold, and the Poisson bracket of functions on this space gives a Lie algebra of observables. Multisymplectic geometry describes an $n$-dimensional field theory using a phase space that is an ' $n$-plectic manifold': a finite-dimensional manifold equipped with a closed nondegenerate $(n+1)$-form. Here we consider the case $n=2$. For any 2-plectic manifold, we construct a Lie 2-algebra of observables. We then explain how this Lie 2-algebra can be used to describe the dynamics of a classical bosonic string. Just as the presence of an electromagnetic field affects the symplectic structure for a charged point particle, the presence of a $B$ field affects the 2-plectic structure for the string.
\end{abstract}

\section{Introduction}

It is becoming clear that string theory can be viewed as a 'categorification' of particle physics, in which familiar algebraic and geometrical structures based in set theory are replaced by their category-theoretic analogues. The basic idea is simple. While a classical particle has a position nicely modelled by an element of a set, namely a point in space:

the position of a classical string is better modelled by a morphism in a category, namely an unparametrized path in space:

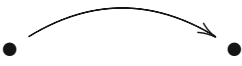


Similarly, while particles have worldlines in spacetime, which can be thought of as morphisms, strings have worldsheets, which can be thought of as 2-morphisms.

So far this viewpoint has been most fruitful in studying the string-theoretic generalizations of gauge theory [8]. The first clue was the $B$ field in string theory. The electromagnetic field contributes to the change in phase of a charged particle as it moves through spacetime. This field is locally described by a 1 -form $A$, which we integrate along the particle's worldline to compute a phase change. The $B$ field contributes to the phase change of a charged string in a similar way: it is locally described by a 2 -form, which we integrate over a string's worldsheet. When we seek a global description suitable for nontrivial spacetime topologies, the electromagnetic field is better thought of as a connection on a U(1) bundle. Similarly, the $B$-field is globally described by a connection on the categorified version of a U(1) bundle, namely a U(1) gerbe [11,18, 19,37].

Later it was found that connections on nonabelian gerbes also play a role in string theory $[1,2,10]$. Nonabelian gerbes are a special case of 2-bundles: roughly speaking, bundles with smooth categories rather than smooth manifolds as fibers [9]. To understand connections on general 2-bundles, it was necessary to categorify the concepts of Lie group and Lie algebra, obtaining the notions of Lie 2-group [6,7] and Lie 2-algebra $[5,30]$.

Still more recently, iterated categorification has become important in the generalizations of gauge theory suited to higher-dimensional membranes [16,32,33]. It is clear by now that to understand the behavior of such membranes, we need to study $n$-connections on $n$-bundles: that is, structures analogous to connections that live on things like bundles with smooth $(n-1)$-categories as fibers. In the very simplest case - a topologically trivial $n$-bundle with the simplest nontrivial abelian ' $n$-group' playing the role of gauge group - an $n$-connection is just an $n$-form on the base space. In a straightforward generalization of electromagnetism, the integral of this $n$-form over the membrane's 'worldvolume' contributes to its change in phase.

Given all this, we should expect that as we look deeper into the analogy between point particles, strings, and higher-dimensional membranes, we should find more examples of categorification. Perhaps the most obvious place to look is symplectic geometry. The reason is that symplectic geometry also uses a connection on a U(1) bundle to describe the change of phase of a point particle.

The simplest example is a free particle moving in some Lorentzian manifold $M$ representing spacetime. If we keep track of the particle's momentum as well as its position, it traces out a path in the cotangent bundle $X=T^{*} M$. The cotangent bundle is equipped with a canonical 1 -form $\alpha$, and we can integrate $\alpha$ over this path to determine the particle's change of phase. This is not the historical reason why $X$ is called a 'phase space', but the coincidence is a happy one.

The exterior derivative $\omega=d \alpha$ plays an important role in this story. First, by Stokes' theorem, the integral of this 2-form over any disc in $X$ measures the change of phase of a particle as it moves around the boundary of the disc. A deeper fact is that $\omega$ is a symplectic structure: that is, not only closed but nondegenerate. This lets us take any smooth function $F: X \rightarrow \mathbb{R}$ and find a unique vector field $v_{F}$ such that

$$
\iota_{v_{F}} \omega=-d F,
$$

where $\iota$ stands for interior product, and the minus sign is just a historically popular convention. We should think of $F$ as an 'observable' assigning a number to any state of the particle. In good situations, the vector field $v_{F}$ will generate a one-parameter group of symmetries of $X$ : that is, a flow preserving $\omega$. So, the symplectic nature of $\omega$ guarantees that observables give rise to symmetries. Moreover, by measuring how rapidly one 
observable changes under the one-parameter group of symmetries generated by another, we obtain a binary operation on observables, the Poisson bracket:

$$
\{F, G\}=L_{v_{F}} G
$$

where $L$ stands for Lie derivative. This makes the vector space of observables into a Lie algebra.

Symplectic geometry generalizes this idea by replacing $T^{*} M$ with a more general phase space $X$. We could simply let $X$ be any manifold equipped with a 1 -form $\alpha$ such that $\omega=d \alpha$ is symplectic. However, a 1 -form is the same as a connection on a trivial $\mathrm{U}(1)$ bundle, and $\omega$ is then the curvature of this connection. Since physics is local, it makes more sense to equip $X$ with a locally trivial U(1) bundle $P \rightarrow X$, together with a connection on $P$ whose curvature 2 -form $\omega$ is symplectic. This is the basic context for geometric quantization.

We can study symplectic geometry without assuming that the symplectic 2-form $\omega$ is the curvature of a connection on some $U(1)$ bundle. In particular, we still obtain a Lie algebra of observables using the formulas above. But some of the physical meaning of the symplectic structure only reveals itself in the presence of a U(1) bundle: namely, that the integral of $\omega$ over any disc in $X$ measures the change of phase of a particle as it moves around the boundary of this disc. So, in geometric quantization the U(1) bundle is crucial. We can build such a bundle whenever we can lift the de Rham cohomology class $[\omega] \in H^{2}(X, \mathbb{R})$ to an element of the integral cohomology $H^{2}(X, \mathbb{Z})$.

Now let us consider how all this generalizes when we move from point particles to strings. As a first step towards understanding this, let us return to the point particle moving in a spacetime manifold $M$. We have said that the particle's phase changes in a way described by integrating the canonical 1 -form $\alpha$ along its path in $T^{*} M$. However, in the presence of the electromagnetic field there is an additional phase change due to electromagnetism, at least when the particle is charged. To take this into account, we add to $\alpha$ the 1-form $A$ describing the electromagnetic field, pulled back from $M$ to $T^{*} M$. We then redefine the symplectic structure to be $\tilde{\omega}=d(\alpha+A)$. So, electromagnetism affects the symplectic structure on the cotangent bundle of spacetime. A more detailed account of this can be found in the book by Guillemin and Sternberg [21].

This suggests that when we pass from point particles to strings, and the electromagnetic field is replaced by the $B$ field, we should correspondingly adjust our concept of 'symplectic structure'. Instead of a canonical 1-form, we should have some sort of canonical 2-form on phase space, so we can add the $B$ field to this 2 -form. But this in turn suggests that the analogue of the symplectic structure will be a 3 -form!

This raises the puzzle: how can we generalize symplectic geometry with a 3-form replacing the usual 2-form?

Amusingly, the answer is very old: it goes back to the work of DeDonder [15] and Weyl [35] in the 1930s. Their ideas have been more fully developed in the subject called 'multisymplectic geometry'. For an introduction, try for example the papers by Gotay, Isenberg, Marsden and Montgomery [20], Hélein and Kouneiher [22,23], Kijowski [27], and Rovelli [29]. In particular, Gotay et al have already applied multisymplectic geometry to classical string theory. There are various ways to do this. In this Introduction we take a very naive approach, which will be corrected in Sect. 2.

To begin with, note that just as the position and velocity of a point particle in the spacetime $M$ are given by a point in the tangent bundle $T M$, we could try to describe the position and velocity of a string by a point in $\Lambda^{2} T M$ - that is, a point in $M$ together with a tangent bivector. Similarly, just as the position and momentum of a particle are 
given by a point in $T^{*} M$, we could try to describe the position and momentum of a string by a point in $\Lambda^{2} T^{*} M$.

Just as $T^{*} M$ is equipped with a canonical 1-form, the generalized phase space $X=$ $\Lambda^{2} T^{*} M$ is equipped with a canonical 2-form $\alpha$, as described in Example 2 below. The corresponding 3-form $\omega=d \alpha$ is 'multisymplectic', meaning that it is closed and also nondegenerate in the following sense:

$$
\iota_{v} \omega=0 \Rightarrow v=0
$$

for all vector fields $v$. This means that for any 1 -form $F$, there is at most one vector field $v_{F}$ such that

$$
\iota_{v_{F}} \omega=-d F .
$$

This resembles the equation we have already seen in symplectic geometry, which associates symmetries to observables. But there is a difference: now $v_{F}$ may not exist. So, we should consider a 1 -form $F$ on $X$ to be an observable only when there exists a vector field $v_{F}$ satisfying the above equation.

We can then define a Poisson bracket of observables by the usual formula:

$$
\{F, G\}=L_{v_{F}} G
$$

The result is always another observable. But, we do not obtain a Lie algebra of observables, because this Poisson bracket is only antisymmetric up to an exact 1-form. Exact 1 -forms are always observables, but they play a special role, since they give rise to trivial symmetries: if $F$ is exact, $v_{F}=0$.

This suggests that in the stringy analogue of symplectic geometry we should seek, not a Lie algebra of observables, but a Lie 2-algebra of observables - that is, a category resembling a Lie algebra, with observables as objects. In this category two observables $F$ and $G$ will be deemed 'isomorphic' if they differ by an exact 1 -form. This guarantees that they generate the same symmetries: $v_{F}=v_{G}$.

Indeed, such a Lie 2-algebra exists. After reviewing multisymplectic geometry in Sect. 2, we prove in Thm. 5 that for any manifold $X$ equipped with a closed nondegenerate 3 -form $\omega$, there is a Lie 2-algebra for which:

- An object is a 1-form $F$ on $X$ for which there exists a vector field $v_{F}$ with $\iota_{v_{F}} \omega=$ $-d F$.

- A morphism $f: F \rightarrow F^{\prime}$ is a function $f$ such that $F+d f=F^{\prime}$.

- The bracket of objects $F, G$ is $L_{v_{F}} G$.

On a more technical note, this Lie 2-algebra is 'hemistrict' in the sense of Roytenberg [30]. This means that the Jacobi identity holds on the nose, but the skew-symmetry of the bracket holds only up to isomorphism.

In Thm. 6 we construct another Lie 2-algebra with the same objects and morphisms, where the Lie bracket of objects is given instead by $\iota_{v_{G}} \iota_{v_{F}} \omega$. This Lie 2-algebra is 'semistrict', meaning that the bracket is skew-symmetric, but the Jacobi identity holds only up to isomorphism (as suspected by Kanatchikov [25]). In Thm. 7 we show that these two Lie 2-algebras are isomorphic. This may seem surprising at first, but the notion of 'isomorphism' for Lie 2-algebra is sufficiently supple that superficially different Lie 2-algebras - one hemistrict, one semistrict — can be isomorphic.

In Sect. 5, we apply these ideas to the classical bosonic string propagating in Minkowski spacetime. Following standard ideas in multisymplectic geometry, we replace 
$\Lambda^{2} T^{*} M$ with a more sophisticated 2-plectic manifold: the first cojet bundle of the bundle $\Sigma \times M \rightarrow \Sigma$, where $\Sigma$ is a surface parametrizing the string worldsheet. We explain how to derive the equations of motion for the string from a 2-plectic formulation involving this phase space. We describe an observable 1 -form $H$ on this phase space whose corresponding vector field $v_{H}$ generates time evolution. We also describe how the presence of a $B$ field modifies the 2-plectic structure. Finally, we list some open questions in Sect. 6.

\section{Multisymplectic Geometry}

The idea of multisymplectic geometry is simple and beautiful: associated to any $n$ dimensional classical field theory there is a finite-dimensional 'extended phase space' $X$ equipped with a nondegenerate closed $(n+1)$-form $\omega$. When $n=1$, we are back to the classical mechanics of point particles and ordinary symplectic geometry. When $n=2$, the examples include classical bosonic string theory, as explained in Sect. 5.

However, at this point an annoying terminological question intrudes: what do we call multisymplectic geometry for a fixed value of $n$ ? The obvious choice is ' $n$-symplectic geometry', but unfortunately, this term already means something else [13]. So, until a better choice comes along, we will use the term ' $n$-plectic geometry':

Definition 1. An $(n+1)$-form $\omega$ on a $C^{\infty}$ manifold $X$ is multisymplectic, or more specifically an $\boldsymbol{n}$-plectic structure, if it is both closed:

$$
d \omega=0,
$$

and nondegenerate:

$$
\forall v \in T_{x} X, \iota_{v} \omega=0 \Rightarrow v=0
$$

where we use $\iota_{v} \omega$ to stand for the interior product $\omega(v, \cdot, \ldots, \cdot)$. If $\omega$ is an n-plectic form on $X$ we call the pair $(X, \omega)$ a multisymplectic manifold, or $\boldsymbol{n}$-plectic manifold.

The references already provided contain many examples of multisymplectic manifolds. More examples, together with constraints on which manifolds can admit $n$-plectic structures, have been discussed by Cantrijn et al [12] and Ibort [24]. Here we give four well-known examples.

The first example arises in work related to the Wess-Zumino-Witten model and loop groups:

Example 1. If $G$ is a compact simple Lie group, there is a 3-form $\omega$ on $G$ that is invariant under both left and right translations, which is unique up to rescaling. It is given by

$$
\omega\left(v_{1}, v_{2}, v_{3}\right)=\left\langle v_{1},\left[v_{2}, v_{3}\right]\right\rangle
$$

when $v_{i}$ are tangent vectors at the identity of $G$ (that is, elements of the Lie algebra), and $\langle\cdot, \cdot\rangle$ is the Killing form. This makes $(G, \omega)$ into a 2-plectic manifold. 
The second was already mentioned in the Introduction:

Example 2. Suppose $M$ is a smooth manifold, and let $X=\Lambda^{n} T^{*} M$ be the $n^{\text {th }}$ exterior power of the cotangent bundle of $M$. Then there is a canonical $n$-form $\alpha$ on $X$ given as follows:

$$
\alpha\left(v_{1}, \ldots, v_{n}\right)=x\left(d \pi\left(v_{1}\right), \ldots, d \pi\left(v_{n}\right)\right)
$$

where $v_{1}, \ldots v_{n}$ are tangent vectors at the point $x \in X$, and $\pi: X \rightarrow M$ is the projection from the bundle $X$ to the base space $M$. Note that in this formula we are applying the $n$-form $x \in \Lambda^{n} T^{*} M$ to the $n$-tuple of tangent vectors $d \pi\left(v_{i}\right)$ at the point $\pi(x)$. The $(n+1)$-form

$$
\omega=d \alpha
$$

is $n$-plectic.

Indeed, this can be seen by explicit computation. Let $q^{1}, \ldots, q^{d}$ be coordinates on an open set $U \subseteq M$. Then there is a basis of $n$-forms on $U$ given by $d q^{I}=d q^{i_{1}} \wedge \cdots \wedge d q^{i_{n}}$ where $I=\left(i_{1}, \ldots, i_{n}\right)$ ranges over multi-indices of length $n$. Corresponding to these $n$-forms there are fiber coordinates $p_{I}$ which combined with the coordinates $q^{i}$ pulled back from the base give a coordinate system on $\Lambda^{n} T^{*} U$. In these coordinates we have

$$
\alpha=p_{I} d q^{I},
$$

where we follow the Einstein summation convention to sum over repeated multi-indices of length $n$. It follows that

$$
\omega=d p_{I} \wedge d q^{I}
$$

Using this formula one can check that $\omega$ is indeed $n$-plectic.

The next example, involving an $n$-plectic manifold called $\Lambda_{1}^{n} T^{*} E$, may seem like a technical variation on the theme of the previous one. However, it is actually quite significant, since $n$-plectic manifolds of this sort serve as the extended phase spaces for many classical field theories [14,20,29]. In Sect. 5, we use a 2-plectic manifold of this sort as the extended phase space for the classical bosonic string.

Example 3. Let $\pi: E \rightarrow \Sigma$ be a fiber bundle over an $n$-dimensional manifold $\Sigma$. Given a point $y \in E$, a tangent vector $v \in T_{y} E$ is said to be vertical if $d \pi(v)=0$. There is a vector sub-bundle $\Lambda_{1}^{n} T^{*} E$ of the $n$-form bundle $\Lambda^{n} T^{*} E$ whose fiber at $y \in E$ consists of all $\beta \in \Lambda^{n} T_{y}^{*} E$ such that

$$
\iota_{v_{1}} \iota_{v_{2}} \beta=0
$$

for all vertical vectors $v_{1}, v_{2} \in T_{y} E$. Let $i: \Lambda_{1}^{n} T^{*} E \hookrightarrow \Lambda^{n} T^{*} E$ denote the inclusion. Let $\omega=d \alpha$ be the $n$-plectic form defined in Example 2. Then the pullback $i^{*} \omega$ is an $n$-plectic form on $\Lambda_{1}^{n} T^{*} E$.

Again, this can be seen by explicit calculation. In our application to strings, $E$ will be a trivial bundle $E=\Sigma \times M$ over $\Sigma$, and $\Sigma$ will be equipped with a volume form. It is enough to consider this case, because proving that $i^{*} \omega$ is $n$-plectic is a local calculation, and we can always trivialize $E$ and equip $\Sigma$ with a volume form locally. 
Let $q^{1}, \ldots, q^{n}$ be local coordinates on $\Sigma$ and let $u^{1}, \ldots, u^{d}$ be local coordinates on $M$. Then $\Lambda_{1}^{n} T^{*} E$ has a local basis of sections given by $n$-forms of two types: first, the wedge product of all $n$ cotangent vectors of type $d q^{i}$ :

$$
d q^{1} \wedge \cdots \wedge d q^{n}
$$

and second, wedge products of $n-1$ cotangent vectors of type $d q^{i}$ and a single one of type $d u^{a}$ :

$$
d q^{1} \wedge \cdots \wedge \widehat{d q^{i}} \wedge \cdots \wedge d q^{n} \wedge d u^{a}
$$

Here the hat means that we omit the factor of $d q^{i}$. If $y=(x, u) \in \Sigma \times M$, this basis gives an isomorphism

$$
\Lambda_{1}^{n} T_{y}^{*} E \cong \Lambda^{n} T_{x}^{*} \Sigma \oplus \Lambda^{n-1} T_{x}^{*} \Sigma \otimes T_{u}^{*} M .
$$

In calculations to come, it will be better to use the pulled back volume form $\pi^{*}$ vol as a substitute for the coordinate-dependent $n$-form $d q^{1} \wedge \cdots \wedge d q^{n}$ on $E$. This gives another basis of sections of $\Lambda_{1}^{n} T^{*} E$, which by abuse of notation we call

$$
d Q=\pi^{*} \text { vol }
$$

and

$$
d Q_{i}^{a}=\left(\pi^{*} \iota_{\partial / \partial q^{i}} \operatorname{vol}\right) \wedge d u^{a} .
$$

Corresponding to this basis there are local coordinates $P$ and $P_{a}^{i}$ on $\Lambda_{1}^{n} T^{*} E$, which combined with the coordinates $q^{i}$ and $u^{a}$ pulled back from $E$ give a local coordinate system on $\Lambda_{1}^{n} T^{*} E$. In these coordinates we have:

$$
i^{*} \alpha=P d Q+P_{a}^{i} d Q_{i}^{a},
$$

where again we use the Einstein summation convention. It follows that

$$
i^{*} \omega=d P \wedge d Q+d P_{a}^{i} \wedge d Q_{i}^{a} .
$$

Using this formula one can check that $i^{*} \omega$ is indeed $n$-plectic.

The manifold $\Lambda_{1}^{n} T^{*} E$ may seem rather mysterious, but the next example shows that under good conditions it is isomorphic to the 'first cojet bundle' $J^{1} E^{\star}$. A point in the first jet bundle $J^{1} E$ records the value and first derivative of a section of $E$ at some point of the base space $\Sigma$. So, a first-order Lagrangian $\ell$ for a field theory where fields are sections of $E$ is a function $\ell: J^{1} E \rightarrow \mathbb{R} . J^{1} E$ is thus the natural home for the Lagrangian approach to such field theories. Similarly, the first cojet bundle $J^{1} E^{\star}$ is the natural home for the DeDonder-Weyl Hamiltonian approach to field theory. In particular, the isomorphism

$$
J^{1} E^{\star} \cong \Lambda_{1}^{n} T^{*} E
$$

makes the first cojet bundle into an $n$-plectic manifold.

In the classical mechanics of a point particle, we can take $\Sigma=\mathbb{R}$ to represent time and take $M$ to be some manifold representing space. Then $E=\Sigma \times M$ is the total space of a trivial bundle $E \rightarrow \Sigma$, and a section of this bundle describes the path of a 
particle in space. The first jet bundle $J^{1} E$ is the bundle $\mathbb{R} \times T M$ over the 'extended configuration space' $\mathbb{R} \times M$. On the other hand, the first cojet bundle $J^{1} E^{\star}$ is isomorphic as a symplectic manifold to $T^{*}(\mathbb{R} \times M)$. This is the familiar 'extended phase space' for a particle in the space $M$.

For a more relativistic picture, we may instead take $\Sigma=\mathbb{R}$ to be the parameter space for the path of a particle moving in a manifold $M$ representing spacetime. As before, $E=\Sigma \times M$ is the total space of a trivial bundle $E \rightarrow \Sigma$, but now a section of this bundle describes the worldline of a particle in spacetime.

In Sect. 5 we modify this picture a bit further by letting $\Sigma$ be 2-dimensional, so it represents the parameter space for a string moving in $M$. We again let $E=\Sigma \times M$ be the total space of a trivial bundle $E \rightarrow \Sigma$. Now a section of $E$ describes the worldsheet of a string - and as we shall see, the 2-plectic manifold $J^{1} E^{\star}$ serves as a kind of 'extended phase space' for the string.

Example 4. As in the previous example, let $\pi: E \rightarrow \Sigma$ be a fiber bundle with $\operatorname{dim} \Sigma=$ $n$. Let $\Gamma_{x}(E)$ be the set of smooth sections of $E$ defined in some neighborhood of the point $x \in \Sigma$. Given $\phi \in \Gamma_{x}(E)$, let $j_{x}^{1} \phi$ be the equivalence class of sections whose firstorder Taylor expansion agrees with the first-order Taylor expansion of $\phi$ at the point $x$. The set

$$
J^{1} E=\left\{j_{x}^{1} \phi \mid x \in \Sigma, \phi \in \Gamma_{x}(E)\right\}
$$

is a manifold. Moreover, $J^{1} E$ is the total space of a fiber bundle $\pi_{J}: J^{1} E \rightarrow E$, the first jet bundle of $E$, where

$$
\pi_{J}\left(j_{x}^{1} \phi\right)=\phi(x)
$$

To see these facts it suffices to work locally, so suppose $E=\Sigma \times M$. Let $q^{i}$ be local coordinates on $\Sigma$ and let $u^{a}$ be local coordinates on $M$. These give rise to local coordinates on $J^{1} E$ such that the coordinates for a point $j_{x}^{1} \phi \in J^{1} E$ are $\left(q^{i}, u^{a}, u_{i}^{a}\right)$, where:

$$
q^{i}=q^{i}(x), \quad u^{a}=\left(u^{a} \circ \phi\right)(x), \quad u_{i}^{a}=\frac{\partial u^{a} \circ \phi}{\partial q^{i}}(x) .
$$

The projection $\pi_{J}$ sends the point with coordinates $\left(q^{i}, u^{a}, u_{i}^{a}\right)$ to the point with coordinates $\left(q^{i}, u^{a}\right)$, so $\pi_{J}: J^{1} E \rightarrow E$ is indeed a fiber bundle.

Let $y=(x, u) \in E$. The fiber of $J^{1} E$ over $y$ is

$$
J_{y}^{1} E \cong\left\{A: T_{x} \Sigma \rightarrow T_{y} E \mid d \pi \circ A=1\right\}
$$

where 1 is the identity map on $T_{x} \Sigma$. This is not naturally a vector space, but it is an affine space. To see this, note that a difference of two maps $A, A^{\prime}: T_{x} \Sigma \rightarrow T_{y} E$ lying in $J_{y}^{1} E$ is the same as a linear map from $T_{x} \Sigma$ to the space $V_{y} E$ consisting of vertical vectors at the point $y \in E$. Thus $J_{y}^{1} E$ is an affine space modeled on the vector space $T_{x}^{*} \Sigma \otimes V_{y} E$, and $J^{1} E$ is a bundle of affine spaces. (For details, see Saunders [31].)

Let $J_{y}^{1} E^{\star}$ be the affine dual of $J_{y}^{1} E$, that is, the vector space of affine functions from this affine space to $\mathbb{R}$. There is a vector bundle $J^{1} E^{\star}$ over $E$, the first cojet bundle of 
$E$, whose fiber over $y \in E$ is $J_{y}^{1} E^{\star}$. In fact, a volume form on $\Sigma$ determines a vector bundle isomorphism

$$
J^{1} E^{\star} \cong \Lambda_{1}^{n} T^{*} E .
$$

With the help of the $n$-plectic structure on $\Lambda_{1}^{n} T^{*} E$ described in the previous example, this gives an $n$-plectic structure on $J^{1} E^{\star}$.

The above isomorphism has been explained by Cariñena, Crampin, Ibort [14] and Gotay et al [20]. For us it will be enough to describe it when $E$ is a trivial bundle over $\Sigma$, say $E=\Sigma \times M$, and $\Sigma$ is equipped with a volume form, vol. Using this extra structure, in Example 3 we constructed a specific isomorphism

$$
\Lambda_{1}^{n} T_{y}^{*} E \cong \Lambda^{n} T_{x}^{*} \Sigma \oplus \Lambda^{n-1} T_{x}^{*} \Sigma \otimes T_{u}^{*} M
$$

where $y=(x, u) \in \Sigma \times M$. The volume form on $\Sigma$ also determines isomorphisms

$$
\begin{aligned}
\mathbb{R} \stackrel{\sim}{\rightarrow} \Lambda^{n} T_{x}^{*} \Sigma \\
c \mapsto c \operatorname{vol}_{x}
\end{aligned}
$$

and

$$
\begin{aligned}
T_{x} \Sigma & \stackrel{\sim}{\rightarrow} \Lambda^{n-1} T_{x}^{*} \Sigma \\
v & \mapsto \iota_{v} \operatorname{vol}_{x} .
\end{aligned}
$$

We thus obtain an isomorphism

$$
\Lambda_{1}^{n} T_{y}^{*} E \cong \mathbb{R} \oplus T_{x} \Sigma \otimes T_{u}^{*} M .
$$

On the other hand, the trivialization $E=\Sigma \times M$ gives an isomorphism of affine spaces

$$
J_{y}^{1} E \cong T_{x}^{*} \Sigma \otimes T_{u} M
$$

which has the side-effect of making $J_{y}^{1} E$ into a vector space. When an affine space $V$ happens to be a vector space, we have an isomorphism $V^{\star} \cong \mathbb{R} \oplus V^{*}$, since an affine map to $\mathbb{R}$ is a linear map plus a constant. So, we obtain

$$
J_{y}^{1} E^{\star} \cong \mathbb{R} \oplus T_{x} \Sigma \otimes T_{u}^{*} M .
$$

This gives a specific vector bundle isomorphism $J^{1} E^{\star} \cong \Lambda_{1}^{n} T^{*} E$, as desired.

It will be useful to see this isomorphism in terms of local coordinates. We have already described local coordinates $\left(q^{i}, u^{a}, u_{i}^{a}\right)$ on $J^{1} E$. Taking the affine dual of each fiber, we obtain local coordinates $\left(q^{i}, u^{a}, P_{a}^{i}, P\right)$ on $J^{1} E^{\star}$. We described local coordinates with the same names on $\Lambda_{1}^{n} T^{*} E$ in Example 3. In terms of these coordinates, the isomorphism is given simply by

$$
\begin{aligned}
J^{1} E^{\star} & \stackrel{\sim}{\rightarrow} \Lambda_{1}^{n} T^{*} E, \\
\left(q^{i}, u^{a}, P_{a}^{i}, P\right) & \mapsto\left(q^{i}, u^{a}, P_{a}^{i}, P\right) .
\end{aligned}
$$

Using this isomorphism to transport the $(n-1)$-form $i^{*} \alpha$ given by Eq. (1) from $\Lambda_{1}^{n} T^{*} E$ to $J_{1} E^{\star}$, we obtain this differential form:

$$
\theta=P d Q+P_{a}^{i} d Q_{i}^{a}
$$

on $J_{1} E^{\star}$. Differentiating, it follows that

$$
d \theta=d P \wedge d Q+d P_{a}^{i} \wedge d Q_{i}^{a}
$$

is an $n$-plectic structure on $J^{1} E^{\star}$. 


\section{Poisson Brackets}

Next we describe how to generalize Poisson brackets of observables from symplectic geometry to multisymplectic geometry. Ordinary Hamiltonian mechanics corresponds to 1-plectic geometry, and in this case, observables are smooth functions on phase space. In $n$-plectic geometry, observables will be smooth $(n-1)$-forms - but not all of them, only certain 'Hamiltonian' ones:

Definition 2. Let $(X, \omega)$ be an n-plectic manifold. An $(n-1)$-form $F$ on $X$ is Hamiltonian if there exists a vector field $v_{F}$ on $X$ such that

$$
d F=-\iota_{v_{F}} \omega .
$$

We say $v_{F}$ is the Hamiltonian vector field corresponding to $F$. The set of Hamiltonian $(n-1)$ forms on a multisymplectic manifold is a vector space and is denoted as $\operatorname{Ham}(X)$.

The Hamiltonian vector field $v_{F}$ is unique if it exists. However, except for the familiar case $n=1$, there may be $(n-1)$-forms $F$ having no Hamiltonian vector field. The reason is that given an $n$-plectic form $\omega$ on $X$, this map:

$$
\begin{aligned}
T_{x} X & \rightarrow \Lambda^{n} T_{x}^{*} X, \\
v & \mapsto i_{v} \omega
\end{aligned}
$$

is one-to-one, but not necessarily onto unless $n=1$.

The following proposition generalizes Liouville's Theorem:

Proposition 1. If $F \in \operatorname{Ham}(X)$, then the Lie derivative $L_{v_{F}} \omega$ is zero.

Proof. Since $\omega$ is closed, $L_{v_{F}} \omega=d \iota_{v_{F}} \omega=-d d F=0$.

We can define a Poisson bracket of Hamiltonian $(n-1)$-forms in two ways:

Definition 3. Given $F, G \in \operatorname{Ham}(X)$, the hemi-bracket $\{F, G\}_{\mathrm{h}}$ is the $(n-1)$-form given by

$$
\{F, G\}_{\mathrm{h}}=L_{v_{F}} G .
$$

Definition 4. Given $F, G \in \operatorname{Ham}(X)$, the semi-bracket $\{F, G\}_{\mathrm{S}}$ is the $(n-1)$-form given by

$$
\{F, G\}_{\mathrm{s}}=\iota_{v_{G}} \iota_{v_{F}} \omega .
$$

The two brackets agree in the familiar case $n=1$, but in general they differ by an exact form:

Proposition 2. Given $F, G \in \operatorname{Ham}(X)$,

$$
\{F, G\}_{\mathrm{h}}=\{F, G\}_{\mathrm{s}}+d \iota_{v_{F}} G .
$$

Proof. Since $L_{v}=\iota_{v} d+d \iota_{v}$,

$$
\{F, G\}_{\mathrm{h}}=L_{v_{F}} G=\iota_{v_{F}} d G+d \iota_{v_{F}} G=-\iota_{v_{F}} \iota_{v_{G}} \omega+d \iota_{v_{F}} G=\{F, G\}_{\mathrm{s}}+d \iota_{v_{F}} G .
$$


Both brackets have nice properties:

Proposition 3. Let $F, G, H \in \operatorname{Ham}(X)$ and let $v_{F}, v_{G}, v_{H}$ be the respective Hamiltonian vector fields. The hemi-bracket $\{\cdot, \cdot\}_{\mathrm{h}}$ has the following properties:

1. The bracket of Hamiltonian forms is Hamiltonian:

$$
d\{F, G\}_{\mathrm{h}}=-\iota_{\left[v_{F}, v_{G}\right]} \omega,
$$

so in particular we have

$$
v_{\{F, G\}_{\mathrm{h}}}=\left[v_{F}, v_{G}\right] .
$$

2. The bracket is antisymmetric up to an exact form:

$$
\{F, G\}_{\mathrm{h}}+d S_{F, G}=-\{G, F\}_{\mathrm{h}}
$$

with $S_{F, G}=-\left(\iota_{v_{F}} G+\iota_{v_{G}} F\right)$.

3. The bracket satisfies the Jacobi identity:

$$
\left\{F,\{G, H\}_{\mathrm{h}}\right\}_{\mathrm{h}}=\left\{\{F, G\}_{\mathrm{h}}, H\right\}_{\mathrm{h}}+\left\{G,\{F, H\}_{\mathrm{h}}\right\}_{\mathrm{h}} .
$$

Proof. 1. If $F, G \in \operatorname{Ham}(X)$, then $d\{F, G\}_{\mathrm{h}}=-\iota_{\left[v_{F}, v_{G}\right]} \omega-\iota_{v_{G}} L_{v_{F}} \omega$, by the identities relating the Lie derivative, exterior derivative, and interior product. Proposition 1 then implies the desired result.

2. Rewriting the Lie derivative in terms of $d$ and $\iota$ gives

$$
\begin{aligned}
\{F, G\}_{\mathrm{h}}+\{G, F\}_{\mathrm{h}} & =\iota_{v_{G}} \iota_{v_{F}} \omega+\iota_{v_{F}} \iota_{v_{G}} \omega+d\left(\iota_{v_{F}} G+\iota_{v_{G}} F\right) \\
& =-d S_{F, G} .
\end{aligned}
$$

3. The definition of the bracket and Property 1 give

$$
\begin{aligned}
\left\{\{F, G\}_{\mathrm{h}}, H\right\}_{\mathrm{h}}+\left\{G,\{F, H\}_{\mathrm{h}}\right\}_{\mathrm{h}} & =L_{\left[v_{F}, v_{G}\right]} H+L_{v_{G}} L_{v_{F}} H \\
& =L_{v_{F}} L_{v_{G}} H \\
& =\left\{F,\{G, H\}_{\mathrm{h}}\right\}_{\mathrm{h}} .
\end{aligned}
$$

Proposition 4. Let $F, G, H \in \operatorname{Ham}(X)$ and let $v_{F}, v_{G}, v_{H}$ be the respective Hamiltonian vector fields. The semi-bracket $\{\cdot, \cdot\}_{\mathrm{S}}$ has the following properties:

1. The bracket of Hamiltonian forms is Hamiltonian:

$$
d\{F, G\}_{\mathrm{s}}=-\iota_{\left[v_{F}, v_{G}\right]} \omega,
$$

so in particular we have

$$
v_{\{F, G\}_{\mathrm{s}}}=\left[v_{F}, v_{G}\right] .
$$

2. The bracket is antisymmetric:

$$
\{F, G\}_{\mathrm{s}}=-\{G, F\}_{\mathrm{s}} .
$$

3. The bracket satisfies the Jacobi identity up to an exact form:

$$
\left\{F,\{G, H\}_{\mathrm{S}}\right\}_{\mathrm{S}}+d J_{F, G, H}=\left\{\{F, G\}_{\mathrm{S}}, H\right\}_{\mathrm{s}}+\left\{G,\{F, H\}_{\mathrm{s}}\right\}_{\mathrm{S}}
$$

with $J_{F, G, H}=-\iota_{v_{F}} \iota_{v_{G}} \iota_{v_{H}} \omega$. 
Proof. 1. Proposition 2 and Prop. 3 imply $d\{F, G\}_{\mathrm{S}}=-\iota_{\left[v_{F}, v_{G}\right]} \omega$.

2. The conclusion follows from the antisymmetry of $\omega$.

3. First, note that antisymmetry implies $\{F, G\}_{\mathrm{s}}=-\iota_{v_{F}} \iota_{v_{G}} \omega=\iota_{v_{F}} d G$. Hence

$$
\begin{aligned}
& \left\{F,\{G, H\}_{\mathrm{s}}\right\}_{\mathrm{s}}=\iota_{v_{F}} d\{G, H\}_{\mathrm{s}}=\iota_{v_{F}} d \iota_{v_{G}} d H, \\
& \left\{\{F, G\}_{\mathrm{s}}, H\right\}_{\mathrm{s}}=\iota_{v_{\{F, G\}_{\mathrm{S}}}} d H=\iota_{\left[v_{F}, v_{G}\right]} d H, \\
& \left\{G,\{F, H\}_{\mathrm{s}}\right\}_{\mathrm{s}}=\left\{G, \iota_{v_{F}} d H\right\}_{\mathrm{s}}=\iota_{v_{G}} d \iota_{v_{F}} d H .
\end{aligned}
$$

The commutator of the Lie derivative and interior product:

$$
\iota_{\left[v_{F}, v_{G}\right]}=L_{v_{F}} \iota_{v_{G}}-\iota_{v_{G}} L_{v_{F}},
$$

and Weil's identity:

$$
L_{v_{F}}=d \iota_{v_{F}}+\iota_{v_{F}} d, \quad L_{v_{G}}=d \iota_{v_{G}}+\iota_{v_{G}} d
$$

imply

$$
\begin{aligned}
\{F & \left.,\{G, H\}_{\mathrm{s}}\right\}_{\mathrm{s}}-\left\{\{F, G\}_{\mathrm{s}}, H\right\}_{\mathrm{s}}-\left\{G,\{F, H\}_{\mathrm{s}}\right\}_{\mathrm{s}} \\
& =\left(-\iota_{\left[v_{F}, v_{G}\right]}+\iota_{v_{F}} d \iota_{v_{G}}-\iota_{v_{G}} d \iota_{v_{F}}\right) d H \\
& =\left(\iota_{v_{G}} L_{v_{F}}-L_{v_{F}} \iota_{v_{G}}+\iota_{v_{F}} d \iota_{v_{G}}-\iota_{v_{G}} d \iota_{v_{F}}\right) d H \\
& =\left(\iota_{v_{G}} \iota_{v_{F}} d-d \iota_{v_{F}} \iota_{v_{G}}\right) d H \\
& =-d \iota_{v_{F}} \iota_{v_{G}} d H=d \iota_{v_{F}} \iota_{v_{G}} \iota_{v_{H}} \omega=-d J_{F, G, H} .
\end{aligned}
$$

In general, neither the hemi-bracket nor the semi-bracket makes Ham $(X)$ into a Lie algebra, since each satisfies one of the Lie algebra laws only up to an exact $(n-1)$-form. The exception is $n=1$, the case of ordinary Hamiltonian mechanics. In this case both brackets equal the usual Poisson bracket. In what follows we consider the case $n=2$.

\section{Lie 2-Algebras}

Next we review the fully general Lie 2-algebras defined by Roytenberg [30], and introduce the Lie 2-algebras of observables in 2-plectic geometry. It will be efficient to work with these using the language of chain complexes. A Lie 2-algebra is a category equipped with structures analogous to those of a Lie algebra. So, to begin with, it is a '2-vector space': a category where the set of objects and the set of morphisms are vector spaces, and all the category operations are linear. However, one can show [5] that a 2-vector space is the same as a 2-term complex: that is, a chain complex of vector spaces that vanishes except in degrees 0 and 1:

$$
L_{0} \stackrel{d}{\leftarrow} L_{1} \stackrel{0}{\leftarrow} 0 \stackrel{0}{\leftarrow} 0 \stackrel{0}{\leftarrow} \cdots .
$$


This lets us define a Lie 2-algebra as a 2-term complex equipped with a bracket operation satisfying the usual Lie algebra laws 'up to coherent chain homotopy'.

In particular, the bracket of 0-chains will be skew-symmetric up to a chain homotopy called the 'alternator':

$$
[x, y]+d S_{x, y}=-[y, x]
$$

while the Jacobi identity will hold up to a chain homotopy called the 'Jacobiator':

$$
[x,[y, z]]+d J_{x, y, z}=[[x, y], z]+[y,[x, z]] .
$$

Furthermore, these chain homotopies need to satisfy some laws of their own. If the alternator vanishes, a Lie 2-algebra is the same as a 2 -term complex made into an ' $L_{\infty^{-}}$algebra' or 'sh Lie algebra' in the sense of Stasheff [28]. Roytenberg introduced more general Lie 2-algebras where the alternator does not vanish.

The definitions to come require a few preliminary explanations. First, we use the familiar tensor product of chain complexes:

$$
(L \otimes M)_{i}=\bigoplus_{j+k=i} L_{j} \otimes M_{k}
$$

With this, the tensor product of 2-term complexes is a 3-term complex. Previous work on Lie 2-algebras used a 'truncated' tensor product of 2-term complexes, which gives another 2-term complex [5,30]. But since this makes no difference to anything we do here, we shall use the familiar tensor product.

Second, given chain complexes $L$ and $M$, we use

$$
\sigma: L \otimes M \rightarrow M \otimes L
$$

to denote the usual 'switch' map with signs included:

$$
\sigma(x \otimes y)=(-1)^{\operatorname{deg} x \operatorname{deg} y} y \otimes x .
$$

Third, given 0-chains $x, y$ and a 1-chain $T$ with $y=x+d T$, we write

$$
T: x \rightarrow y \text {. }
$$

We also write 1: $x \rightarrow x$ in the case where the 1-chain $T$ vanishes, and write $S T: x \rightarrow z$ for the 1-chain $S+T$, where $T: x \rightarrow y$ and $S: y \rightarrow z$. This notation alludes to how a 2-term chain complex can be thought of as a category.

In this notation, the alternator in a Lie 2-algebra $L$ gives a 1-chain

$$
S_{x, y}:[x, y] \rightarrow-[y, x]
$$

for every pair of 0 -chains $x, y$, and the Jacobiator gives a 1-chain

$$
J_{x, y, z}:[x,[y, z]] \rightarrow[[x, y], z]+[y,[x, z]]
$$

for every triple of 0 -chains $x, y, z$. 
Definition 5. A Lie 2-algebra is a 2-term chain complex of vector spaces $L=\left(L_{0} \stackrel{d}{\leftarrow}\right.$ $\left.L_{1}\right)$ equipped with the following structure:

- a chain map $[\cdot, \cdot]: L \otimes L \rightarrow L$ called the bracket;

- a chain homotopy

$$
S:[\cdot, \cdot] \Rightarrow-[\cdot, \cdot] \circ \sigma
$$

called the alternator;

- a chain homotopy

$$
J:[\cdot,[\cdot, \cdot]] \Rightarrow[[\cdot, \cdot], \cdot]+[\cdot,[\cdot, \cdot]] \circ(\sigma \otimes 1)
$$

called the Jacobiator.

In addition, the following diagrams are required to commute:

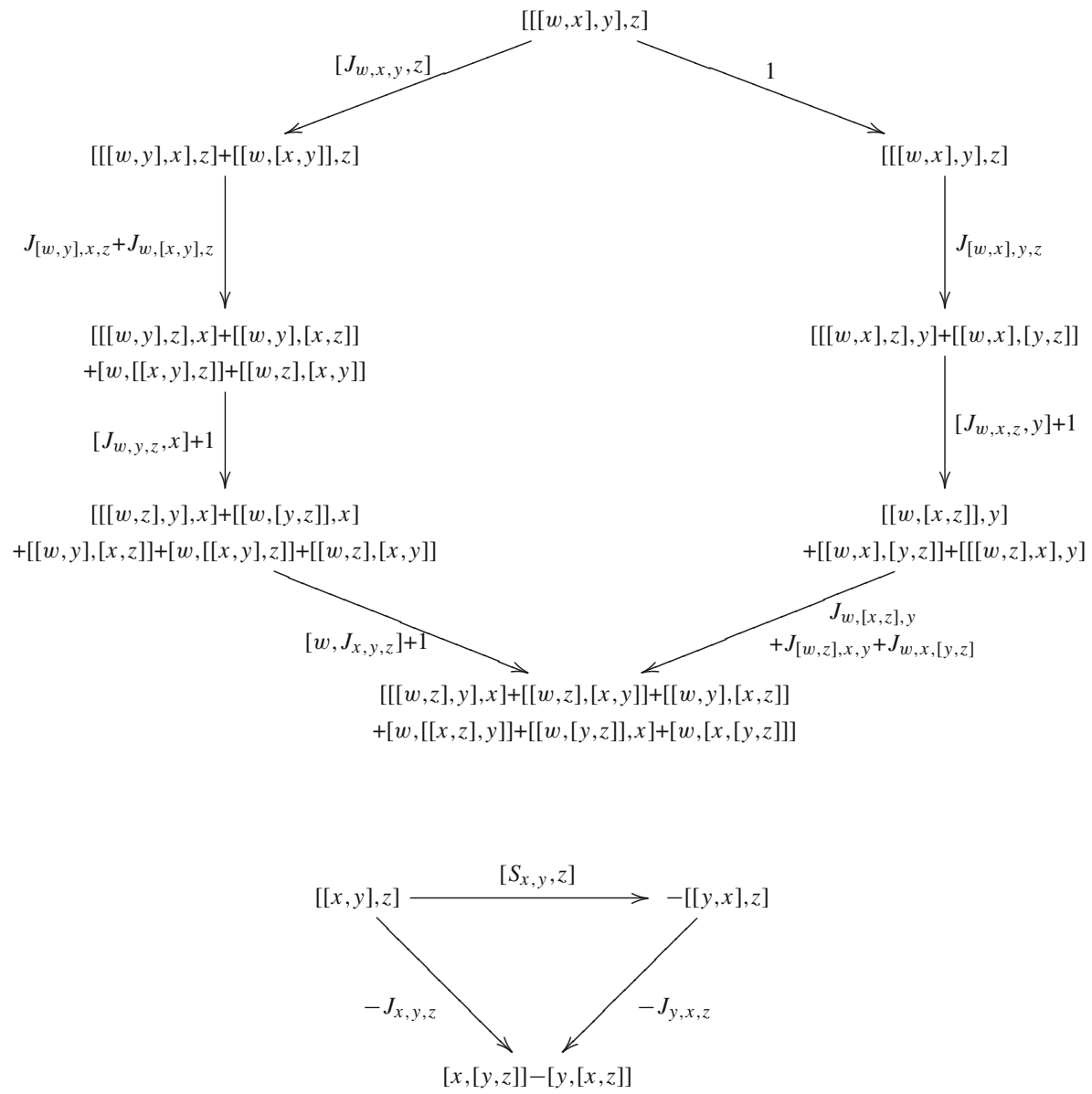



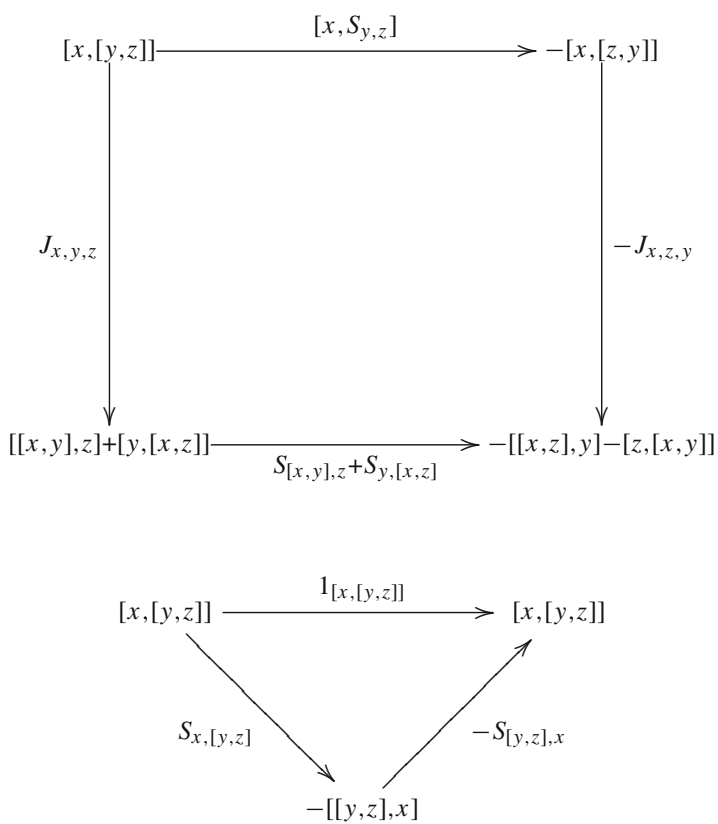

Definition 6. A Lie 2-algebra for which the Jacobiator is the identity chain homotopy is called hemistrict. One for which the alternator is the identity chain homotopy is called semistrict.

When the alternator is the identity, the Jacobiator $J_{x, y, z}$ is antisymmetric as a function of $x, y$ and $z$, so the semistrict Lie 2-algebras defined here match those of Baez and Crans [5].

Now suppose that $(X, \omega)$ is a 2-plectic manifold. We shall construct two Lie 2-algebras associated to $(X, \omega)$ : one hemistrict and one semistrict. Then we shall prove these are isomorphic. Both these Lie 2-algebras have the same underlying 2-term complex, namely:

$$
L=\operatorname{Ham}(X) \stackrel{d}{\leftarrow} C^{\infty}(X) \stackrel{0}{\leftarrow} 0 \stackrel{0}{\leftarrow} 0 \stackrel{0}{\leftarrow} \cdots,
$$

where $d$ is the usual exterior derivative of functions. To see that this chain complex is well-defined, note that any exact form is Hamiltonian, with 0 as its Hamiltonian vector field.

The hemistrict Lie 2-algebra comes with a bracket called the hemi-bracket:

$$
\{\cdot, \cdot\}_{\mathrm{h}}: L \otimes L \rightarrow L
$$

In degree 0, the hemi-bracket is given as in Def. 3:

$$
\{F, G\}_{\mathrm{h}}=L_{v_{F}} G \text {. }
$$

In degree 1 , it is given by:

$$
\{F, f\}_{\mathrm{h}}=L_{v_{F}} f, \quad\{f, F\}_{\mathrm{h}}=0 .
$$


In degree 2 , we necessarily have

$$
\{f, g\}_{\mathrm{h}}=0 \text {. }
$$

Here $F, G \in \operatorname{Ham}(X)$, while $f, g \in C^{\infty}(X)$.

To see that the hemi-bracket is in fact a chain map, it suffices to check it on hemibrackets of degree 1 :

$$
d\{F, f\}_{\mathrm{h}}=d\left(L_{v_{F}} f\right)=L_{v_{F}} d f=\{F, d f\}_{\mathrm{h}}
$$

and

$$
d\{f, F\}_{\mathrm{h}}=0=L_{v_{d f}} F=\{d f, F\}_{\mathrm{h}},
$$

since the Hamiltonian vector field corresponding to an exact 1-form is zero.

Theorem 5. If $(X, \omega)$ is a 2-plectic manifold, there is a hemistrict Lie 2-algebra $L(X, \omega)_{\mathrm{h}}$ where:

- the space of 0-chains is $\operatorname{Ham}(X)$,

- the space of 1-chains is $C^{\infty}(X)$,

- the differential is the exterior derivative $d: C^{\infty}(X) \rightarrow \operatorname{Ham}(X)$,

- the bracket is $\{\cdot, \cdot\}_{\mathrm{h}}$,

- the alternator is the bilinear map $S$ : $\operatorname{Ham}(X) \times \operatorname{Ham}(X) \rightarrow C^{\infty}(X)$ defined by $S_{F, G}=-\left(\iota_{v_{F}} G+\iota_{v_{G}} F\right)$, and

- the Jacobiator is the identity, hence given by the trilinear map $J: \operatorname{Ham}(X) \times$ $\operatorname{Ham}(X) \times \operatorname{Ham}(X) \rightarrow C^{\infty}(X)$ with $J_{F, G, H}=0$.

Proof. That $S$ is a chain homotopy with the right source and target follows from Prop. 3 and the fact that:

$\{F, f\}_{\mathrm{h}}+\{g, G\}_{\mathrm{h}}+S_{F, d f}+S_{d g, G}=L_{v_{F}} f-\iota_{v_{F}} d f-\iota_{v_{G}} d g=-\{f, F\}_{\mathrm{h}}-\{G, g\}_{\mathrm{h}}$.

Proposition 3 also says that the Jacobi identity holds. The following equations then imply that $J$ is also a chain homotopy with the right source and target:

$$
\begin{aligned}
& \left\{F,\{G, f\}_{\mathrm{h}}\right\}_{\mathrm{h}}=\left\{\{F, G\}_{\mathrm{h}}, f\right\}_{\mathrm{h}}+\left\{G,\{F, f\}_{\mathrm{h}}\right\}_{\mathrm{h}}, \\
& \left\{F,\{f, G\}_{\mathrm{h}}\right\}_{\mathrm{h}}=\left\{\{F, f\}_{\mathrm{h}}, G\right\}_{\mathrm{h}}=\left\{f,\{F, G\}_{\mathrm{h}}\right\}_{\mathrm{h}}=0, \\
& \left\{f,\{F, G\}_{\mathrm{h}}\right\}_{\mathrm{h}}=\left\{\{f, F\}_{\mathrm{h}}, G\right\}_{\mathrm{h}}=\left\{F,\{f, G\}_{\mathrm{h}}\right\}_{\mathrm{h}}=0 .
\end{aligned}
$$

So, we just need to check that the Lie 2-algebra axioms hold. The first two diagrams commute since each edge is the identity. The commutativity of the third diagram is shown as follows:

$$
\begin{aligned}
S_{\{F, G\}_{\mathrm{h}}, H}+S_{G,\{F, H\}_{\mathrm{h}}} & =-\iota_{\left[v_{F}, v_{G}\right]} H-\iota_{v_{H}}\{F, G\}_{\mathrm{h}}-\iota_{v_{G}}\{F, H\}_{\mathrm{h}}-\iota_{\left[v_{F}, v_{H}\right]} G \\
& =L_{v_{F}}\left(-\iota_{v_{G}} H-\iota_{v_{H}} G\right) \\
& =\left\{F, S_{G, H}\right\}_{\mathrm{h}} .
\end{aligned}
$$

The last diagram says that

$$
S_{F,\{G, H\}_{\mathrm{h}}}-S_{\{G, H\}_{\mathrm{h}}, F}=0,
$$

and this follows from the fact that the alternator is symmetric: $S_{F, G}=S_{G, F}$. 
Next we make $L$ into a semistrict Lie 2-algebra. For this, we use a chain map called the semi-bracket:

$$
\{\cdot, \cdot\}_{\mathrm{S}}: L \otimes L \rightarrow L
$$

In degree 0, the semi-bracket is given as in Def. 4:

$$
\{F, G\}_{\mathrm{s}}=\iota_{v_{G}} \iota_{v_{F}} \omega .
$$

In degrees 1 and 2, we set it equal to zero:

$$
\{F, f\}_{\mathrm{s}}=0, \quad\{f, F\}_{\mathrm{s}}=0, \quad\{f, g\}_{\mathrm{s}}=0 .
$$

Theorem 6. If $(X, \omega)$ is a 2-plectic manifold, there is a semistrict Lie 2-algebra $L(X, \omega)_{\mathrm{S}}$ where:

- the space of 0-chains is $\operatorname{Ham}(X)$,

- the space of 1-chains is $C^{\infty}(X)$,

- the differential is the exterior derivative $d: C^{\infty}(X) \rightarrow \operatorname{Ham}(X)$,

- the bracket is $\{\cdot, \cdot\}_{\mathrm{s}}$,

- the alternator is the identity, hence given by the bilinear map $S: \operatorname{Ham}(X) \times$ $\operatorname{Ham}(X) \rightarrow C^{\infty}(X)$ with $S_{F, G}=0$, and

- the Jacobiator is the trilinear map $J$ : $\operatorname{Ham}(X) \times \operatorname{Ham}(X) \times \operatorname{Ham}(X) \rightarrow C^{\infty}(X)$ defined by $J_{F, G, H}=-\iota_{v_{F}} \iota_{v_{G}} \iota_{v_{H}} \omega$.

Proof. We note from Prop. 4 that the semi-bracket is antisymmetric. Since both $S$ and the bracket in degree 1 are zero, the alternator defined above is a chain homotopy with the right source and target. It follows from Prop. 4 and that the Hamiltonian vector field of an exact 1-form is zero that the Jacobiator is also a chain homotopy with the desired source and target. So again, we just need to check that the Lie 2-algebra axioms hold. The following identities can be checked by simple calculation, and the commutativity of the first diagram follows:

$$
\begin{gathered}
J_{\{K, F\}_{\mathrm{s}}, G, H}=J_{\{H, K\}_{\mathrm{s}}, F, G}-J_{\{F, H\}_{\mathrm{s}}, G, K}-L_{v_{G}} J_{K, F, H}, \\
L_{v_{G}} J_{K, F, H}=J_{\{G, K\}_{\mathrm{s}}, F, H}+J_{K,\{G, F\}_{\mathrm{s}}, H}+J_{K, F,\{G, H\}_{\mathrm{s}}} .
\end{gathered}
$$

Since the Jacobiator is antisymmetric and the alternator is the identity, the second and third diagrams commute as well. The fourth diagram commutes because all the edges are identity morphisms.

Definition 7. Given Lie 2-algebras $L$ and $L^{\prime}$ with bracket, alternator and Jacobiator $[\cdot, \cdot], S, J$ and $[\cdot, \cdot]^{\prime}, S^{\prime}, J^{\prime}$ respectively, a homomorphism from $L$ to $L^{\prime}$ consists of:

- a chain map $\phi: L \rightarrow L^{\prime}$, and

- a chain homotopy $\Phi:[\cdot, \cdot]^{\prime} \circ(\phi \otimes \phi) \Rightarrow \phi \circ[\cdot, \cdot]$,

such that the following diagrams commute: 

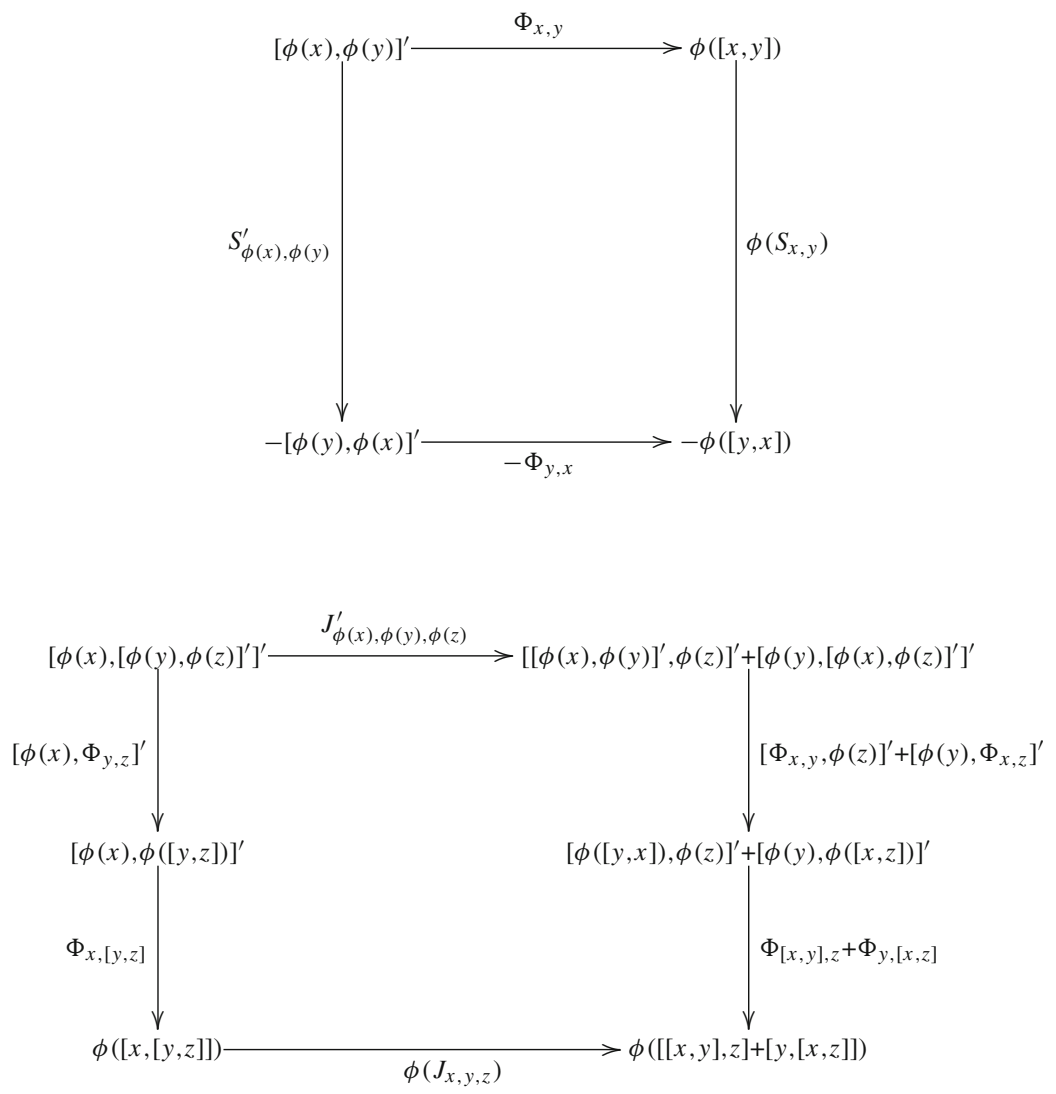

Roytenberg explains how to compose Lie 2-algebra homomorphisms [30], and we say a Lie 2-algebra homomorphism with an inverse is an isomorphism.

Theorem 7. $L(X, \omega)_{\mathrm{h}}$ and $L(X, \omega)_{\mathrm{s}}$ are isomorphic as Lie 2-algebras.

Proof. We show that the identity chain maps with appropriate chain homotopies define Lie 2-algebra homomorphisms and that their composites are the respective identity homomorphisms. There is a homomorphism $\phi: L(X, \omega)_{\mathrm{h}} \rightarrow L(X, \omega)_{\mathrm{s}}$ with the identity chain map and the chain homotopy given by $\Phi_{F, G}=\iota_{v_{F}} G$. That this is a chain homotopy follows from the bracket relation $\{F, G\}_{\mathrm{S}}+d\left(\iota_{v_{F}} G\right)=\{F, G\}_{\mathrm{h}}$ noted in Prop. 2 together with the equations

$$
\{F, f\}_{\mathrm{s}}+\iota_{v_{F}} d f=\{F, f\}_{\mathrm{h}}, \quad\{f, F\}_{\mathrm{s}}=\{f, F\}_{\mathrm{h}}=\iota_{v_{d f}} F=0 .
$$

We check that the two diagrams in the definition of a Lie 2-algebra homomorphism commute. Noting that the chain map is the identity, the commutativity of the first diagram is easily checked by recalling that $S_{F, G}=-\left(\iota_{v_{G}} F+\iota_{v_{F}} G\right)$ and that $S_{F, G}^{\prime}$ is the identity. Noting that any edge given by the bracket for $L(X, \omega)_{\mathrm{s}}$ in degree 1 is the identity and that $J_{F, G, H}$ is the identity, to check the commutativity of the second diagram we only need to perform the following calculation: 


$$
\begin{aligned}
& J_{F, G, H}^{\prime}+\Phi_{\{F, G\}_{\mathrm{h}}, H}+\Phi_{G,\{F, H\}_{\mathrm{h}}}-\Phi_{F,\{G, H\}_{\mathrm{h}}} \\
& \quad=-\iota_{v_{F}} \iota_{v_{G}} \iota_{v_{H}} \omega+\iota_{\left[v_{F}, v_{G}\right]} H+\iota_{v_{G}} L_{v_{F}} H-\iota_{v_{F}} L_{v_{G}} H \\
& =\iota_{v_{F}} L_{v_{G}} H-\iota_{v_{F}} d \iota_{v_{G}} H+\iota_{\left[v_{F}, v_{G}\right]} H+\iota_{v_{G}} L_{v_{F}} H-\iota_{v_{F}} L_{v_{G}} H \\
& =-\iota_{v_{F}} d \iota_{v_{G}} H+\iota_{\left[v_{F}, v_{G}\right]} H+\iota_{v_{G}} L_{v_{F}} H \\
& =-\iota_{v_{F}} d \iota_{v_{G}} H+L_{v_{F}} \iota_{v_{G}} H-\iota_{v_{G}} L_{v_{F}} H+\iota_{v_{G}} L_{v_{F}} H \\
& =-\iota_{v_{F}} d \iota_{v_{G}} H+L_{v_{F}} \iota_{v_{G}} H \\
& =d \iota_{v_{F}} \iota_{v_{G}} H-L_{v_{F}} \iota_{v_{G}} H+L_{v_{F}} \iota_{v_{G}} H \\
& =d \iota_{v_{F}} \iota_{v_{G}} H \\
& =0 .
\end{aligned}
$$

\section{The Classical Bosonic String}

The bosonic string is a theory of maps $\phi: \Sigma \rightarrow M$ where $\Sigma$ is a surface and $M$ is some manifold representing spacetime. For simplicity we will only consider the case where $\Sigma$ is the cylinder $\mathbb{R} \times S^{1}$ and $M$ is $d$-dimensional Minkowski spacetime, $\mathbb{R}^{1, d-1}$. A solution of the classical bosonic string is then a map $\phi: \Sigma \rightarrow M$ which is a critical point of the area subject to certain boundary conditions.

Equivalently, by exploiting symmetries in the variational problem, one can describe solutions $\phi$ by equipping $\mathbb{R} \times S^{1}$ with its standard Minkowski metric and then solving the $1+1$ dimensional field theory specified by the Lagrangian density

$$
\ell=\frac{1}{2} g^{i j} \eta_{a b} \frac{\partial \phi^{a}}{\partial q^{i}} \frac{\partial \phi^{b}}{\partial q^{j}} .
$$

Here $q^{i}(i=0,1)$ are standard coordinates on $\mathbb{R} \times S^{1}$ and $g=\operatorname{diag}(1,-1)$ is the Minkowski metric on $\mathbb{R} \times S^{1}$, while $\phi^{a}$ are the coordinates of the map $\phi$ in $\mathbb{R}^{1, d-1}$ and $\eta=\operatorname{diag}(1,-1, \ldots,-1)$ is the Minkowski metric on $\mathbb{R}^{1, d-1}$. We use the Einstein summation convention to sum over repeated indices. The corresponding Euler-Lagrange equation is just a version of the wave equation:

$$
g^{i j} \partial_{i} \partial_{j} \phi^{a}=0
$$

We next describe this theory using multisymplectic geometry following the approach of Hélein [22]. (The work of Gotay et al [20] focuses instead on the Polyakov approach, where the metric on $\Sigma$ is taken as an independent variable.)

The space $E=\Sigma \times M$ can be thought of as a trivial bundle over $\Sigma$, and the graph of a function $\phi: \Sigma \rightarrow M$ is a smooth section of $E$. We write the coordinates of a point $(x, u) \in E$ as $\left(q^{i}, u^{a}\right)$. Let $J^{1} E \rightarrow E$ be the first jet bundle of $E$. As explained in Example 4, since $E$ is trivial we may regard $J^{1} E$ as a vector bundle whose fiber over $(x, u) \in E$ is $T_{x}^{*} \Sigma \otimes T_{u} M$. The Lagrangian density for the string can be defined as a smooth function on $J^{1} E$ :

$$
\ell=\frac{1}{2} g^{i j} \eta_{a b} u_{i}^{a} u_{j}^{b}
$$

which depends in this example only on the fiber coordinates $u_{i}^{a}$. 
Let $J^{1} E^{*} \rightarrow E$ be the vector bundle dual to $J^{1} E$. The fiber of $J^{1} E^{*}$ over $(x, u) \in E$ is $T_{x} \Sigma \otimes T_{u}^{*} M$. From the Lagrangian $\ell: J^{1} E \rightarrow \mathbb{R}$, the 'de Donder-Weyl Hamiltonian' $h: J^{1} E^{*} \rightarrow \mathbb{R}$ can be constructed via a Legendre transform. It is given as follows:

$$
\begin{aligned}
h & =p_{a}^{i} u_{i}^{a}-\ell \\
& =\frac{1}{2} \eta^{a b} g_{i j} p_{a}^{i} p_{b}^{j},
\end{aligned}
$$

where $u_{i}^{a}$ are defined implicitly by $p_{a}^{i}=\partial \ell / \partial u_{i}^{a}$, and $p_{a}^{i}$ are coordinates on the fiber $T_{x} \Sigma \otimes T_{u}^{*} M$. Note that $h$ differs from the standard (non-covariant) Hamiltonian density $\varepsilon$ for a field theory:

$$
\begin{aligned}
\varepsilon & =p_{a}^{0} u_{0}^{a}-\ell \\
& =\frac{1}{2} \eta^{a b}\left(p_{a}^{0} p_{b}^{0}+p_{a}^{1} p_{b}^{1}\right) .
\end{aligned}
$$

Let $\phi$ be a section of $E$ and let $\pi$ be a smooth section of $J^{1} E^{*}$ restricted to $\phi(\Sigma)$ with fiber coordinates $\pi_{a}^{i}$. It is then straightforward to show that $\phi$ is a solution of the Euler-Lagrange equations if and only if $\phi$ and $\pi$ satisfy the following system of equations:

$$
\begin{aligned}
& \frac{\partial \pi_{a}^{i}}{\partial q^{i}}=-\left.\frac{\partial h}{\partial u^{a}}\right|_{u=\phi, p=\pi}, \\
& \frac{\partial \phi^{a}}{\partial q^{i}}=\left.\frac{\partial h}{\partial p_{a}^{i}}\right|_{u=\phi, p=\pi} \cdot
\end{aligned}
$$

This system of equations is a generalization of Hamilton's equations for a classical point particle.

As explained in Example 4 and the preceding discussion, the extended phase space for the string is the first cojet bundle $J^{1} E^{\star}$, and this space is equipped with a canonical 2-form $\theta$ whose exterior derivative $\omega=d \theta$ is a 2-plectic structure. Using the isomorphism

$$
J^{1} E^{\star} \cong J^{1} E^{*} \times \mathbb{R}
$$

a point in $J^{1} E^{\star}$ gets coordinates $\left(q^{i}, u^{a}, p_{a}^{i}, e\right)$. In terms of these coordinates,

$$
\theta=e d q^{0} \wedge d q^{1}+\left(p_{a}^{0} d u^{a} \wedge d q^{1}-p_{a}^{1} d u^{a} \wedge d q^{0}\right)
$$

The 2-plectic structure on $J^{1} E^{\star}$ is thus

$$
\omega=d e \wedge d q^{0} \wedge d q^{1}+\left(d p_{a}^{0} \wedge d u^{a} \wedge d q^{1}-d p_{a}^{1} \wedge d u^{a} \wedge d q^{0}\right) .
$$

So, the variable $e$ may be considered as 'canonically conjugate' to the area form $d q^{0} \wedge$ $d q^{1}$.

As before, let $\phi$ be a section of $E$ and let $\pi$ be a smooth section of $J^{1} E^{*}$ restricted to $\phi(\Sigma)$. Consider the submanifold $S \subset J^{1} E^{\star}$ with coordinates:

$$
\left(q^{i}, \phi^{a}\left(q^{j}\right), \pi_{a}^{i}\left(q^{j}\right),-h\right)
$$


Note that $S$ is constructed from $\phi, \pi$ and from the constraint $e+h=0$. This constraint is analogous to the one that is used in finding constant energy solutions in the extended phase space approach to classical mechanics. At each point in $S$, a tangent bivector $v=v_{0} \wedge v_{1}$ can be defined as

$$
\begin{aligned}
& v_{0}=\frac{\partial}{\partial q^{0}}+\frac{\partial \phi^{a}}{\partial q^{0}} \frac{\partial}{\partial u^{a}}+\frac{\partial \pi_{a}^{i}}{\partial q^{0}} \frac{\partial}{\partial p_{a}^{i}} \\
& v_{1}=\frac{\partial}{\partial q^{1}}+\frac{\partial \phi^{a}}{\partial q^{1}} \frac{\partial}{\partial u^{a}}+\frac{\partial \pi_{a}^{i}}{\partial q^{1}} \frac{\partial}{\partial p_{a}^{i}} .
\end{aligned}
$$

Explicit computation reveals that the submanifold $S$ is generated by solutions to Hamilton's equations if and only if

$$
\omega\left(v_{0}, v_{1}, \cdot\right)=0
$$

Quite generally, infinitesimal symmetries of the 2-form $\theta$ give rise to Hamiltonian 1-forms that generate these symmetries. For example, symmetry under time evolution lets us define a Hamiltonian. Consider the Lie derivative of $\theta$ along the coordinate vector field $\partial / \partial q^{0}$ :

$$
\begin{aligned}
& L_{\partial / \partial q^{0}} \theta=d \iota_{\partial / \partial q^{0}} \theta+\iota_{\partial / \partial q^{0} \omega} \\
& =d\left(e d q^{1}+p_{a}^{1} d u^{a}\right)-\left(d e \wedge d q^{1}+d p_{a}^{1} \wedge d u^{a}\right) \\
& =0 \text {. }
\end{aligned}
$$

Hence $\theta$ is invariant with respect to infinitesimal displacements along the $q^{0}$ coordinate. If we define a 1 -form $H$ by

$$
\begin{aligned}
H & =-\iota_{\partial / \partial q^{0} \theta} \\
& =-e d q^{1}-p_{a}^{1} d u^{a},
\end{aligned}
$$

then $d H=\iota_{\partial / \partial q^{0}} \omega$. Hence $H$ is a Hamiltonian 1-form, and the Hamiltonian vector field $v_{H}$ describes time evolution.

One may wonder how this Hamiltonian 1-form $H$ is related to the usual concept of energy. To understand this, consider the solution submanifold $S$ as defined above. Let $S_{\tau} \subset S$ be a 1-dimensional curve on $S$ at constant 'time' $q^{0}=\tau$. Denote the restriction of $H$ to $S_{\tau}$ as $H_{\tau}$. A computation yields

$$
\begin{aligned}
H_{\tau} & =h d q^{1}-\pi_{a}^{1} d \phi^{a} \\
& =\frac{1}{2} \eta^{a b} g_{i j} \pi_{a}^{i} \pi_{b}^{j} d q^{1}-\pi_{a}^{1} d \phi^{a} .
\end{aligned}
$$

On $S_{\tau}, d q^{0}=0$. Hence $d \phi^{a}=\frac{\partial \phi^{a}}{\partial q^{1}} d q^{1}$. Since $\phi$ satisfies Eq. (13), we also have:

$$
\begin{gathered}
\pi_{a}^{0}=\eta_{a b} \frac{\partial \phi^{b}}{\partial q^{0}}, \\
\pi_{a}^{1}=-\eta_{a b} \frac{\partial \phi^{b}}{\partial q^{1}} .
\end{gathered}
$$


The expression for $H_{\tau}$ thus becomes:

$$
\begin{aligned}
H_{\tau} & =\frac{1}{2} \eta^{a b}\left(\pi_{a}^{0} \pi_{b}^{0}+\pi_{a}^{1} \pi_{b}^{1}\right) d q^{1} \\
& =\frac{1}{2} \eta_{a b}\left(\frac{\partial \phi^{a}}{\partial q^{0}} \frac{\partial \phi^{b}}{\partial q^{0}}+\frac{\partial \phi^{a}}{\partial q^{1}} \frac{\partial \phi^{b}}{\partial q^{1}}\right) d q^{1} \\
& =\varepsilon d q^{1} .
\end{aligned}
$$

Hence $H_{\tau}$ is the Hamiltonian 1-form that corresponds to the energy density of the string at $\tau$, and the total energy of the string at $q^{0}=\tau$ is simply:

$$
\int_{S_{\tau}} H_{\tau}
$$

So, the usual concept of energy is compatible with the concept of energy as a Hamiltonian 1-form in the Lie 2-algebra of observables for the string.

We next consider a scenario in which the string is coupled to a $B$ field. We fix a 2-form $B$ on $M$. By pulling back $d B$ along the projection $p: J^{1} E^{\star} \rightarrow M$ and adding it to the 2-plectic form $\omega$, we obtain a modified 2-plectic form $\tilde{\omega}$ on $J^{1} E^{\star}$ :

$$
\tilde{\omega}=\omega+p^{*} d B
$$

In coordinates:

$$
p^{*} d B=d\left(B_{b c} d u^{b} \wedge d u^{c}\right)=\frac{\partial B_{b c}}{\partial u^{a}} d u^{a} \wedge d u^{b} \wedge d u^{c} .
$$

It is straightforward to show that $\tilde{\omega}$ is indeed 2-plectic.

We now determine the equations of motion for the string coming from the modified 2-plectic structure $\tilde{\omega}$. As before, we consider the submanifold $S$ defined above. We emphasize that we have not changed $h$ : it is still the de Donder-Weyl Hamiltonian for the free string. Requiring $\tilde{\omega}\left(v_{0}, v_{1}, \cdot\right)=0$ implies

$$
\omega\left(v_{0}, v_{1}, \cdot\right)+p^{*} d B\left(v_{0}, v_{1}, \cdot\right)=0 .
$$

Let

$$
J^{b c}=\frac{\partial \phi^{b}}{\partial q^{0}} \frac{\partial \phi^{c}}{\partial q^{1}}-\frac{\partial \phi^{b}}{\partial q^{1}} \frac{\partial \phi^{c}}{\partial q^{0}}
$$

and

$$
F_{b c d}=\frac{\partial B_{c d}}{\partial u^{b}}+\frac{\partial B_{d b}}{\partial u^{c}}+\frac{\partial B_{b c}}{\partial u^{d}} .
$$

It follows that

$$
\left(p^{*} d B\right)\left(v_{0}, v_{1}, \cdot\right)=J^{b c} F_{b c d} d u^{d},
$$

which implies that $\phi$ obeys the following equations:

$$
g^{i j} \partial_{i} \partial_{j} \phi^{a}=\eta^{a d} J^{b c} F_{b c d} .
$$


These equations, familiar from the work of Kalb and Ramond [26], are precisely the Euler-Lagrange equations derived from a Lagrangian density $\tilde{\ell}$ that includes a $B$ field term:

$$
\tilde{\ell}=\ell+J^{a b} B_{a b}
$$

So, adding the pullback of $d B$ to $\omega$ modifies the 2-plectic structure in precisely the right way to give the correct equations of motion for a string coupled to a $B$ field. This generalizes the usual story for point particles coupled to electromagnetism [21].

\section{Conclusions}

The work presented here raises many questions. Here are four obvious ones:

- Does an $n$-plectic manifold give rise to a Lie $n$-algebra when $n>2$ ? There is not yet a definition of weak or hemistrict Lie $n$-algebras for $n>2$, but a semistrict Lie $n$-algebra is just an $n$-term chain complex equipped with the structure of an $L_{\infty}$-algebra. So, it would be easiest to start by considering a generalization of the semi-bracket, and see if this can be used to construct a semistrict Lie $n$-algebra.

- Does the Lie 2-algebra of observables in 2-plectic geometry extend to something like a Poisson algebra? It is far from clear how to define a product for Hamiltonian 1-forms, and the usual product of a Hamiltonian 1-form and a smooth function is not Hamiltonian.

- The based loop space $\Omega X$ of a manifold $X$ equipped with a closed $(n+1)$-form $\omega$ is an infinite-dimensional manifold equipped with a closed $n$-form $\eta$ defined 'by transgression' as follows:

$$
\eta\left(v_{1}, \ldots, v_{n}\right)=\int_{0}^{2 \pi} \omega\left(\gamma^{\prime}(\sigma), v_{1}(\gamma(\sigma)), \ldots, v_{n}(\gamma(\sigma)) d \sigma,\right.
$$

where $v_{i}$ are tangent vectors at the loop $\gamma \in \Omega X$ and $v_{i}(\gamma(\sigma))$ are the corresponding tangent vectors at the point $\gamma(\sigma) \in X$. Even when $\omega$ is $n$-plectic, $\eta$ is rarely $(n-1)$-plectic. However when $X=G$ is a compact simple Lie group equipped with the 2-plectic structure of Example 1, $\eta$ becomes symplectic after adding an exact form. The interplay between the 2-plectic structure on $G$ and the symplectic structure on $\Omega G$ plays an important role in the theory relating the Wess-ZuminoWitten model, central extensions of the loop group $\Omega G$, gerbes on $G$ and the string 2-groups $\operatorname{String}_{k}(G)$ [6]. It would be nice to have a more general theory whereby the loop space of an $n$-plectic manifold became an $(n-1)$-plectic manifold.

- When a symplectic structure $\omega$ on a manifold $X$ defines an integral class in $H^{2}(X, \mathbb{R})$, there is a $U(1)$ bundle over $X$ equipped with a connection whose curvature is $\omega$. As mentioned in the Introduction, this plays a fundamental role in the geometric quantization of $X$. Similarly, when a 2-plectic structure $\omega$ on a manifold $X$ defines an integral class in $H^{3}(X, \mathbb{R})$, there is a $\mathrm{U}(1)$ gerbe over $X$ equipped with a connection whose curvature is $\omega[11]$. Is there an analogue of geometric quantization that applies in this case?

Following the ideas of Freed [17], we might hope that geometrically quantizing this gerbe will give a '2-Hilbert space' of states. However, Freed's work only treats Schrödinger quantization, and that only in the special case where the resulting 2-Hilbert space is finite-dimensional. Finite-dimensional 2-Hilbert spaces are by 
now well-understood [3], but the infinite-dimensional ones are still being developed $[4,36]$. Geometric quantization for gerbes is an even greater challenge. However, we expect the problem of geometrically quantizing a U(1) gerbe on $X$ to be closely related to the better-understood problem of geometrically quantizing the corresponding $\mathrm{U}(1)$ bundle on the loop space of $X$.

Acknowledgements. We thank Urs Schreiber, Allen Knutson and Dmitry Roytenberg for corrections and helpful conversations. This work was partially supported by a grant from the Foundational Questions Institute.

Open Access This article is distributed under the terms of the Creative Commons Attribution Noncommercial License which permits any noncommercial use, distribution, and reproduction in any medium, provided the original author(s) and source are credited.

\section{References}

1. Aschieri, P., Cantini, L., Jurčo, B.: Nonabelian bundle gerbes, their differential geometry and gauge theory. Commun. Math. Phys. 254, 367-400 (2005)

2. Aschieri, P., Jurčo, B.: Gerbes, M5-brane anomalies and $E_{8}$ gauge theory. J. High Energy Phys. 10, 068 (2004)

3. Baez, J.: Higher-dimensional algebra II: 2-Hilbert spaces. Adv. Math. 127, 125-189 (1997)

4. Baez, J., Baratin, A., Freidel, L., Wise, D.: Representations of 2-groups on higher Hilbert spaces. http:// arxiv.org/abs/0812.4969v1, 2008

5. Baez, J., Crans, A.: Higher-dimensional algebra VI: Lie 2-algebras. Theory Appl. Categ. 12, 492-528 (2004)

6. Baez, J., Crans, A., Stevenson, D., Schreiber, U.: From loop groups to 2-groups. Homology, Homotopy Appl. 9, 101-135 (2007)

7. Baez, J., Lauda, A.: Higher-dimensional algebra V: 2-groups. Theory Appl. Categ. 12, 423-491 (2004)

8. Baez, J., Schreiber, U.: Higher gauge theory. In: Davydov, A. et al (eds.) Categories in Algebra, Geometry and Mathematical Physics. Contemp. Math. 431, Providence, RI: Amer. Math. Soc., 2007, pp. 7-30

9. Bartels, T.: Higher gauge theory: 2-bundles. http://arxiv.org/abs/math/0410328v3, 2006

10. Breen, L., Messing, W.: Differential geometry of gerbes. Adv. Math. 198, 732-846 (2005)

11. Brylinski, J.-L.: Loop Spaces, Characteristic Classes and Geometric Quantization. Boston: Birkhauser, 1993

12. Cantrijn, F., Ibort, A., De Leon, M.: On the geometry of multisymplectic manifolds. J. Austral. Math. Soc. Ser. A 66, 303-330 (1999)

13. Cartin, D.: Generalized symplectic manifolds. http://arxiv.org/abs/dg-ga/9710027v2, 1997

14. Cariñena, J.F., Crampin, M., Ibort, L.A.: On the multisymplectic formalism for first order field theories. Diff. Geom. Appl. 1, 345-374 (1991)

15. DeDonder, T.: Theorie Invariantive du Calcul des Variations. Paris: Gauthier-Villars, 1935

16. Diaconescu, E., Moore, G., Freed, D.: The $M$-theory 3-form and $E_{8}$ gauge theory. In: Miller, H.R., Ravenel D.C. (eds.), Elliptic Cohomology. London Math. Soc. Lecture Note Ser. 342, Cambridge: Cambridge Univ. Press, 2007, pp. 44-88

17. Freed, D.: Higher algebraic structures and quantization. Commun. Math. Phys. 159, 343-398 (1994)

18. Freed, D.: Dirac charge quantization and generalized differential cohomology. In: Yau, S.-T. ed., Surveys in Differential Geometry VII, Somerville, MA: International Press, 2000, pp. 129-194

19. Freed, D., Witten, E.: Anomalies in string theory with D-branes. Asian J. Math. 3, 819-851 (1999)

20. Gotay, M., Isenberg, J., Marsden, J., Montgomery, R.: Momentum maps and classical relativistic fields. Part I: covariant field theory. http://arxiv.org/abs/physics/9801019v2, 2004

21. Guillemin, V., Sternberg, S.: Symplectic Techniques in Physics. Cambridge: Cambridge U. Press, 1984

22. Hélein, F.: Hamiltonian formalisms for multidimensional calculus of variations and perturbation theory. In: Bahri, A. et al, eds., Noncompact Problems at the Intersection of Geometry, Providence, RI: Amer. Math. Soc., 2001, pp. 127-148

23. Hélein, F., Kouneiher, J.: The notion of observable in the covariant Hamiltonian formalism for the calculus of variations with several variables. Adv. Theor. Math. Phys. 8, 735-777 (2004)

24. Ibort, A.: Multisymplectic geometry: generic and exceptional. In: Grácia, X. et al, eds. Proceedings of the IX Fall Workshop on Geometry and Physics, Vilanova i la Geltrú, 2000. Publicaciones de la RSME 3, Madrid: Real Sociedad Matemática Española, 2001, pp. 79-88

25. Kanatchikov, I.: Canonical structure of classical field theory in the polymomentum phase space. Rep. Math. Phys. 41, 49-90 (1998) 
26. Kalb, M., Ramond, P.: Classical direct interstring action. Phys. Rev. D 9, 2273-2284 (1974)

27. Kijowski, J.: A finite-dimensional canonical formalism in the classical field theory. Commun. Math. Phys. 30, 99-128 (1973)

28. Lada, T., Stasheff, J.: Introduction to sh Lie algebras for physicists. Int. J. Theor. Phys. 32, 1087-1103 (1993)

29. Rovelli, C.: Covariant Hamiltonian formalism for field theory: Hamilton-Jacobi equation on the space $G$. http://arxiv.org/abs/gr-qc/0207043v2, 2002

30. Roytenberg, D.: On weak Lie 2-algebras. In: Kielanowski, P. et al, eds, XXVI Workshop on Geometrical Methods in Physics. AIP Conference Proceedings 956, Melville: American Institute of Physics, 2007, pp. 180-198

31. Saunders, D.J.: The Geometry of Jet Bundles. London Math. Soc. Lecture Note Ser. 142, Cambridge: Cambridge U. Press, 1989

32. Sati, H., Schreiber, U., Stasheff, J.: $L_{\infty}$-algebra connections and applications to String- and ChernSimons $n$-transport. http://arxiv.org/abs/0801.3480v2, 2008

33. Sati, H., Schreiber, U., Stasheff, J.: Fivebrane structures. http://arxiv.org/abs/0805.0564v3, 2009

34. Schreiber, U.: From Loop Space Mechanics to Nonabelian Strings. Ph.D. thesis, Universität DuisburgEssen (2005); http://arxiv.org/abs/hep-th/0509163v1, 2005

35. Weyl, H.: Geodesic fields in the calculus of variation for multiple integrals. Ann. Math. 36, 607-629 (1935)

36. Yetter, D.: Measurable categories. Appl. Cat. Str. 13, 469-500 (2005)

37. Zunger, Y.: $p$-Gerbes and extended objects in string theory. http://arxiv.org/abs/hep-th/0002074v2, 2000

Communicated by P.T. Chruściel 